OPEN ACCESS

Edited by:

Fabio Grizzi,

Humanitas Clinical and Research

Center, Italy

Reviewed by:

Emmanuel S. Antonarakis,

Johns Hopkins Sidney Kimmel

Comprehensive Cancer Center, USA

Sanja Štifter,

University of Rijeka, Croatia

*Correspondence:

David J. VanderWeele, Department of Medicine, University of Chicago, 5841 S. Maryland Avenue, MC 2115, Chicago, IL 60637, USA djvanderweele@gmail.com

Specialty section:

This article was submitted to Genitourinary Oncology, a section of

the journal Frontiers in Oncology

Received: 29 May 2015 Accepted: 08 July 2015 Published: 22 July 2015

Citation:

VanderWeele DJ, Paner GP,

Fleming GF and Szmulewitz RZ (2015) Sustained complete response to cytotoxic therapy and the PARP inhibitor veliparib in metastatic castration-resistant prostate

cancer - a case report.

Front. Oncol. 5:169.

doi: 10.3389/fonc.2015.00169

\section{Sustained complete response to cytotoxic therapy and the PARP inhibitor veliparib in metastatic castration-resistant prostate cancer - a case report}

\author{
David J. VanderWeele ${ }^{1 *}$, Gladell P. Paner ${ }^{2}$, Gini F. Fleming ${ }^{1}$ and Russell Z. Szmulewitz ${ }^{1}$ \\ ${ }^{1}$ Department of Medicine, University of Chicago, Chicago, IL, USA, ${ }^{2}$ Department of Pathology, University of Chicago, \\ Chicago, IL, USA
}

Solid tumors harboring BRCA1 or BRCA2 mutations have been shown to respond to PARP inhibitors. These responses are partial and transient. In this case report, we describe an 82-year-old male with poorly differentiated prostate cancer with metastases to the lung, liver, abdomen, and bowel. Molecular testing demonstrated alterations in BRCA2, ERG, and TP53. Based on this result, he was enrolled in a therapeutic trial and received carboplatin, gemcitabine, and veliparib, to which he had a partial response. He continued to respond while on veliparib maintenance alone, and after 38 cycles he had a sustained complete response. A sustained complete response to PARP inhibitor-based therapy has not previously been described for prostate cancer. This case suggests that cytotoxic therapy in combination with PARP inhibitors may yield exceptional responses, and molecular studies may help guide patient selection for these therapies.

Keywords: prostate cancer, PARP inhibitor, veliparib, BRCA2, ERG, TP53, complete response

\section{Introduction}

Though genetic alterations serve as a biomarker to guide the use of targeted therapy for many solid tumors, this is rarely employed for prostate cancer. Given the important role of the androgen receptor (AR) in prostate cancer (1), AR-targeted therapies are the backbone of therapy for almost all patients and no specific genetic test is warranted. Emerging data suggest, however, that for some patients specific genetic derangements may predict response to additional therapies. Preclinical data indicate that tumors that harbor an ETS family rearrangement, such as TMPRSS2-ERG, the most frequent cancer rearrangement, may be responsive to PARP inhibitors (2). Those with a neuroendocrine phenotype typically harbor amplification or overexpression of AURKA or MYCN and are hypothesized to respond to an AURKA inhibitor (3). Clinical trials are underway to evaluate the use of PARP inhibitors (NCT01576172) and AURKA inhibitors (NCT01799278) based on genetic features.

Men who are carriers of BRCA2 mutations are at higher risk of developing prostate cancer than non-carriers, and when they are diagnosed with prostate cancer, it tends to be with higher grade, more aggressive disease (4-6). Evidence from prostate cancer and other tumor types has shown efficacy of PARP inhibitors for tumors with BRCA1 or BRCA2 alterations (7-9), but without complete responses. Preclinical data from the TP53-altered prostate cancer line PC3 have demonstrated synergy between veliparib and cytotoxic therapies (10), and those with alterations 
in ATM, another DNA-damage response gene, have also been reported to respond to PARP inhibitors (11).

We present here a patient with an aggressive prostate cancer with visceral metastases harboring a BRCA2 deletion and TMPRSS2-ERG rearrangement, along with TP53 alteration. Based on his BRCA2 deletion, he was enrolled in a clinical trial and treated with carboplatin, gemcitabine, and the PARP inhibitor veliparib. He had a complete response to therapy, and continues on veliparib maintenance therapy 30 months after enrolling in the trial, with no evidence of disease. To our knowledge, this is the first report of complete response to PARP inhibitor-based therapy for a patient with prostate cancer.

\section{Case Report}

The patient, an 82-year-old man, underwent a surgical procedure at age 61 for treatment of urinary retention due to benign prostatic hypertrophy. Twelve years later, he developed similar symptoms, and a prostate biopsy was performed, showing conventional adenocarcinoma of the prostate, Gleason score $4+3=7$. He underwent external beam radiation therapy, and his PSA nadired at $0.5 \mathrm{ng} / \mathrm{ml}$ but rose to $13.5 \mathrm{ng} / \mathrm{ml} 3$ years after radiation therapy. He began androgen deprivation therapy (ADT) with a luteinizing hormone-releasing hormone analog. Despite this, over the next few years he had multiple surgical procedures for continued urinary retention.

A transurethral resection of the prostate performed 6 years after radiation therapy showed sarcomatoid prostatic adenocarcinoma involving the prostatic urethra and bladder neck. Due to persistent hematuria and lower urinary tract symptoms, the patient underwent a salvage cystoprostatectomy with ileal conduit and descending loop colostomy. Pathology revealed high-grade prostatic adenocarcinoma with extensive sarcomatoid and focal neuroendocrine differentiation diffusely involving the prostate and extending into the seminal vesicles, urinary bladder wall including mucosa and large bowel wall, pT4N0 with 20 regional lymph nodes negative for malignancy (Figure 1). His PSA at the time of cystoprostatectomy was $<0.05 \mathrm{ng} / \mathrm{ml}$.

The patient continued on ADT following cystoprostatectomy but developed recurrence of his disease, with radiographically detected masses within the pelvis and lungs 10 months after his surgery. His PSA remained undetectable. An endoscopic biopsy of a colonic mass showed poorly differentiated carcinoma invading colonic mucosa. The tumor cells exhibited similar morphologic features to those in the cystoprostatectomy specimen. He remained minimally symptomatic and was managed conservatively. Six months after his recurrence, he developed worsening rectal bleeding. Sigmoidoscopy showed progression of his metastatic disease in his rectum. Imaging confirmed bowel wall thickening and showed other pelvic disease; progression of his lung metastases, largest measuring $3.5 \mathrm{~cm}$; and new liver metastases, the largest $3.1 \times 3.0 \mathrm{~cm}$ (Figure $2 \mathrm{~A}$ ).

Tumor tissue from the patient's cystoprostatectomy was sent to Foundation One for genetic testing. Three genomic alterations were identified: BRCA2 loss, TMPRSS2-ERG fusion, and TP53 F270V. The BRCA2 loss and TMPRSS2-ERG translocation both suggested that he may have increased sensitivity to a PARP

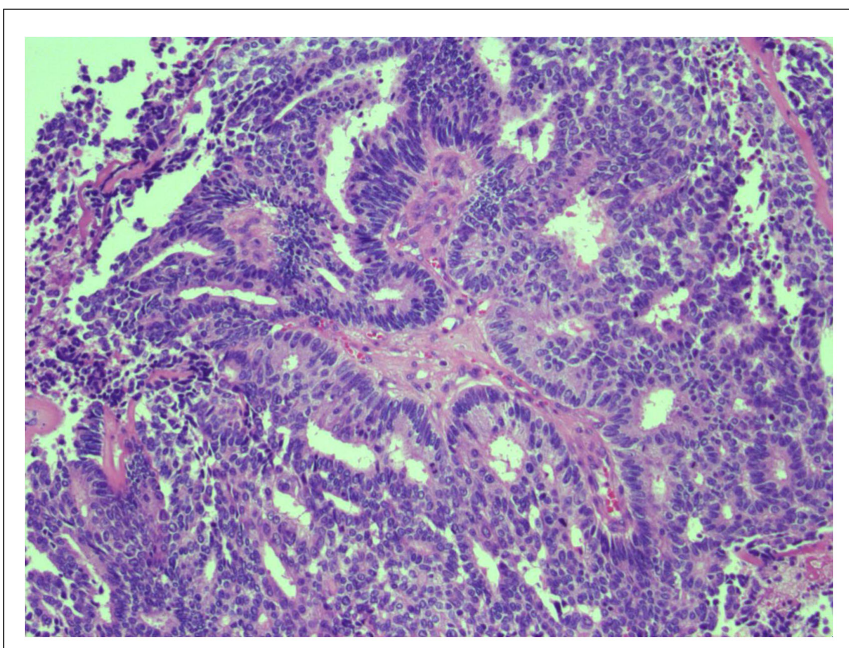

FIGURE 1 | H \& E stain of poorly differentiated prostate cancer. Representative image of high-grade prostate carcinoma from the cystoprostatectomy specimen exhibiting cribriform pattern and cell necrosis.

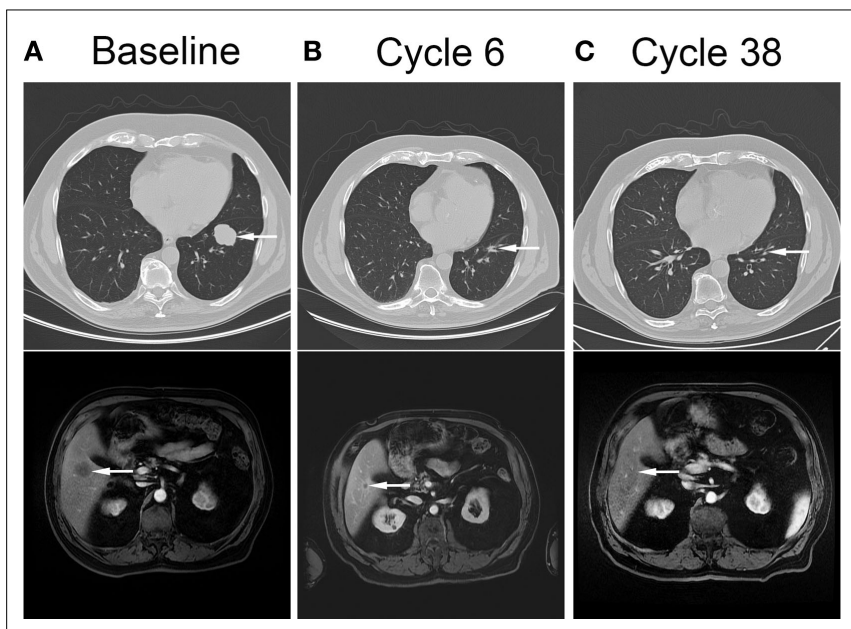

FIGURE 2 | Complete resolution of metastatic disease in response to cytotoxic therapy and veliparib. Computed tomography images showing pulmonary (top panel, arrow) and liver (bottom panel, arrow) metastases at baseline (A), after completion of cytotoxic therapy and veliparib (B), and after 38 cycles of veliparib maintenance therapy (C).

inhibitor, and he provided consent for and was enrolled in an Internal Review Board-approved phase I clinical trial for patients with documented BRCA1/BRCA2 mutations (NCT01063816, IRB 09267) consisting of six 21-day cycles of carboplatin $\mathrm{AUC}=4$ day 1 , gemcitabine $800 \mathrm{mg} / \mathrm{m}^{2}$ days 1 and 8 , and veliparib $250 \mathrm{mg}$ PO BID daily, to be followed by veliparib $250 \mathrm{mg}$ PO BID maintenance therapy. The patient's course was complicated by diarrhea, thrombocytopenia, anemia requiring transfusion, and fatigue. Veliparib was held in cycle 2 for thrombocytopenia, and restarted with cycle 3 at $210 \mathrm{mg}$ PO BID. Gemcitabine was reduced to $400 \mathrm{mg} / \mathrm{m}^{2}$ on cycle 3 day 8 .

Restaging radiographic imaging was performed after three cycles of therapy, which showed a partial response within the liver and lungs. The rectal bleeding had resolved at this time. The patient completed three additional cycles of therapy, dose 
reduced due to thrombocytopenia and consisting of carboplatin $\mathrm{AUC}=4$ day 1 , gemcitabine $400 \mathrm{mg} / \mathrm{m}^{2}$ day 1 , and veliparib $210 \mathrm{mg}$ PO BID. Thrombocytopenia and diarrhea resolved, and anemia and fatigue improved. Repeat staging at the completion of six cycles showed continued decrease in size of lung metastases, stable liver metastases, and resolution of bowel wall thickening (Figure 2B). He started maintenance therapy with veliparib $210 \mathrm{mg}$ PO BID and continued to have improvement in his metastases. Ten months after completion of therapy, he had complete resolution of all sites of metastatic disease, and after 38 cycles of therapy continues to have no evidence of disease (Figure 2C). Currently he continues on veliparib therapy with minimal toxicity.

\section{Discussion}

One could consider castration the first molecularly targeted therapy in oncology, reducing activity of $\mathrm{AR}$, which drives prostate cancer growth and proliferation. Since castration was described as effective therapy for prostate cancer seven decades ago, few therapies for prostate cancer have found targets beyond AR. That is beginning to change, however, with therapies targeting PARP and AURKA now in clinical trials for the disease. As the development of these therapies progresses, we need ways to identify which patients will most benefit from these therapies.

We describe here a complete response to veliparib-containing therapy in a patient with BRCA2 deletion, TMPRSS2-ERG translocation, and TP53 mutation. Preclinical and clinical evidence suggest that all three alterations may contribute to the patient's dramatic response to therapy.

Sensitivity of tumors harboring ERG translocations to PARP inhibitors appears to be dependent on expression of $E R G$ and interaction between $E R G$ and DNAPK. Hormone naïve cancers harboring ERG rearrangements typically express ERG protein, whereas those lacking ERG rearrangements do not. For those with lethal, castrate-resistant disease, however, ERG expression is variable despite the presence of ERG translocation (12). Since expression of the PSA gene KLK3 is regulated by $A R$, as is TMPRSS2, the patient's low PSA value suggested that ERG might not be expressed. $E R G$ expression was confirmed, however, with immunohistochemical analysis (Figure 3).

TMPRSS2-ERG is the most frequent genomic alteration described in localized prostate cancer, with $40-50 \%$ of patients harboring this translocation, and others harboring rearrangements involving other ETS family members $(13,14)$. BRCA2 alterations are less common, but those with germline BRCA2 alterations are known to be at high risk of prostate cancer diagnosis and high risk of having aggressive disease. It is likely that those with aggressive disease are more likely to harbor these particular alterations. Indeed, in cases of localized prostate cancer sequenced by The Cancer Genome Atlas project, the percent of patients with ERG fusions, BRCA2 deletion or mutation, or both is 35,4 , and $2 \%$, respectively. A recent report on castrate-resistant prostate cancer found that $7 \%$ of cases have missense or truncating mutations in BRCA2, and an additional $12 \%$ have alterations in other DNA repair/recombination genes associated with response to PARP inhibitors (15). In cases of end-stage prostate cancer sequenced by the University of Michigan (16), the percent of

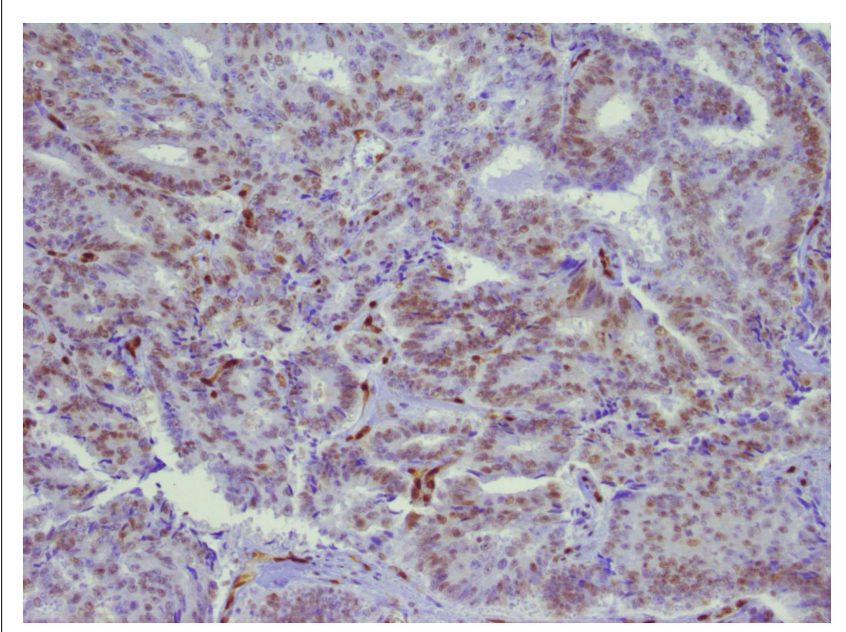

FIGURE 3 | ERG expression in prostate tumor. Immunohistochemical analysis of the primary tumor. ERG expression is indicated by brown chromogen in the nucleus. Blue represents hematoxylin counterstain.

patients with ERG fusions, BRCA2 deletion or mutation, or both is 48,13 , and $8 \%$, respectively. Of those harboring both BRCA2 and ERG alterations, $40 \%$ also harbor a deletion in TP53. Though the percentages are small, given the large number of patients with metastatic prostate cancer, this may represent a large cohort of patients who could be considered for therapy similar to what our patient received. It is possible that alterations in other DNAdamage pathway genes in addition to TP53 would sensitize to PARP inhibitors. The hypothesis that patients with similar known genetic alterations will benefit from a similar treatment regimen needs to be tested in the setting of a prospective clinical trial.

It is unknown if all the components of this therapeutic regimen were necessary in order for this patient to achieve his therapeutic success, as he received carboplatin and gemcitabine in addition to veliparib. However, his disease continued to respond to veliparib alone without achieving a complete response until 10 months after completion of cytotoxic chemotherapy, supporting the notion that there was additional benefit to the veliparib. Although it is conceivable that the patient could have responded to the PARP inhibitor alone (8), other data suggest synergy with cytotoxic therapy (17-21). The optimal duration of treatment is also unknown in this case. The patient has had a sustained complete response 32 cycles after completion of chemotherapy, on veliparib alone. It is unknown if discontinuing veliparib would result in disease recurrence or continued durable response.

Intentionally designed, targeted therapies offer much hope for the treatment of advanced malignancy but often fail to meet expectations. Better combinations of therapy, better biomarkers, or both are needed to help guide therapy decisions. Exploration of the molecular characteristics of those with exceptional responses to therapy is an important tool in improving the use of the therapies we have at hand.

\section{Acknowledgments}

Support for NCT01063816 was provided by Abbvie. 


\section{References}

1. Huggins C, Hodges CV. Studies on prostatic cancer. I. The effect of castration, of estrogen and of androgen injection on serum phosphatases in metastatic carcinoma of the prostate. Cancer Res (1941) 1:293-7.

2. Brenner JC, Ateeq B, Li Y, Yocum AK, Cao Q, Asangani IA, et al. Mechanistic rationale for inhibition of poly(ADP-ribose) polymerase in ETS gene fusionpositive prostate cancer. Cancer Cell (2011) 19:664-78. doi:10.1016/j.ccr.2011. 04.010

3. Beltran H, Rickman DS, Park K, Chae SS, Sboner A, MacDonald TY, et al. Molecular characterization of neuroendocrine prostate cancer and identification of new drug targets. Cancer Discov (2011) 1:487-95. doi:10.1158/ 2159-8290.CD-11-0130

4. Tryggvadóttir L, Vidarsdóttir L, Thorgeirsson T, Jonasson JG, Olafsdóttir EJ, Olafsdóttir GH, et al. Prostate cancer progression and survival in BRCA2 mutation carriers. J Natl Cancer Inst (2007) 99:929-35. doi:10.1093/jnci/djm005

5. Narod SA, Neuhausen S, Vichodez G, Armel S, Lynch HT, Ghadirian P, et al. Rapid progression of prostate cancer in men with a BRCA2 mutation. $\mathrm{Br} J$ Cancer (2008) 99:371-4. doi:10.1038/sj.bjc.6604453

6. Castro E, Goh C, Olmos D, Saunders E, Leongamornlert D, Tymrakiewicz $\mathrm{M}$, et al. Germline BRCA mutations are associated with higher risk of nodal involvement, distant metastasis, and poor survival outcomes in prostate cancer. J Clin Oncol (2013) 31:1748-57. doi:10.1200/JCO.2012.43.1882

7. Fong PC, Boss DS, Yap TA, Tutt A, Wu P, Mergui-Roelvink M, et al. Inhibition of poly(ADP-ribose) polymerase in tumors from BRCA mutation carriers. $N$ Engl J Med (2009) 361:123-34. doi:10.1056/NEJMoa0900212

8. Sandhu SK, Omlin A, Hylands L, Miranda S, Barber LJ, Riisnaes R, et al. Poly (ADP-ribose) polymerase (PARP) inhibitors for the treatment of advanced germline BRCA2 mutant prostate cancer. Ann Oncol (2013) 24:1416-8. doi:10. 1093/annonc/mdt074

9. Kaufman B, Shapira-Frommer R, Schmutzler RK, Audeh MW, Friedlander M, Balmaña J, et al. Olaparib monotherapy in patients with advanced cancer and a germline BRCA1/2 mutation. J Clin Oncol (2014) 33(3):244-50. doi:10.1200/ JCO.2014.56.2728

10. Wu M, Wang X, McGregor N, Pienta KJ, Zhang J. Dynamic regulation of Rad51 by E2F1 and p53 in prostate cancer cells upon drug-induced DNA damage under hypoxia. Mol Pharmacol (2014) 85:866-76. doi:10.1124/mol.113. 090688

11. Mateo J, Hall E, Sandhu S, Omlin AG, Miranda S, Carreira S, et al. Lba20antitumour activity of the Parp inhibitor olaparib in unselected sporadic castration-resistant prostate cancer (crpc) in the Toparp trial. Ann Oncol (2014) 25(Supp 5):v1-41. doi:10.1093/annonc/mdu438.20

12. Udager AM, Shi Y, Tomlins SA, Alva A, Siddiqui J, Cao X, et al. Frequent discordance between ERG gene rearrangement and ERG protein expression in a rapid autopsy cohort of patients with lethal, metastatic, castration-resistant prostate cancer. Prostate (2014) 74(12):1199-208. doi:10.1002/pros.22836

13. Tomlins SA, Rhodes DR, Perner S, Dhanasekaran SM, Mehra R, Sun X-W, et al. Recurrent fusion of TMPRSS2 and ETS transcription factor genes in prostate cancer. Science (2005) 310:644-8. doi:10.1126/science.1117679

14. Rubin MA, Maher CA, Chinnaiyan AM. Common gene rearrangements in prostate cancer. J Clin Oncol (2011) 29:3659-68. doi:10.1200/JCO.2011.35.1916

15. Robinson D, Van Allen EM, Wu Y-M, Schultz N, Lonigro RJ, Mosquera J$\mathrm{M}$, et al. Integrative clinical genomics of advanced prostate cancer. Cell (2015) 161:1215-28. doi:10.1016/j.cell.2015.05.001

16. Grasso CS, Wu Y-M, Robinson DR, Cao X, Dhanasekaran SM, Khan AP, et al The mutational landscape of lethal castration-resistant prostate cancer. Nature (2012) 487:239-43. doi:10.1038/nature11125

17. Donawho CK, Luo Y, Luo Y, Penning TD, Bauch JL, Bouska JJ, et al. ABT888 , an orally active poly(ADP-ribose) polymerase inhibitor that potentiates DNA-damaging agents in preclinical tumor models. Clin Cancer Res (2007) 13:2728-37. doi:10.1158/1078-0432.CCR-06-3039

18. Wu W, Kong Z, Duan X, Zhu H, Li S, Zeng S, et al. Inhibition of PARP1 by small interfering RNA enhances docetaxel activity against human prostate cancer PC3 cells. Biochem Biophys Res Commun (2013) 442:127-32. doi:10.1016/j.bbrc. 2013.11.027

19. Cheng H, Zhang Z, Borczuk A, Powell CA, Balajee AS, Lieberman HB, et al. PARP inhibition selectively increases sensitivity to cisplatin in ERCC1-low non-small cell lung cancer cells. Carcinogenesis (2013) 34:739-49. doi:10.1093/ carcin/bgs 393

20. Shelton JW, Waxweiler TV, Landry J, Gao H, Xu Y, Wang L, et al. In vitro and in vivo enhancement of chemoradiation using the oral PARP inhibitor ABT888 in colorectal cancer cells. Int J Radiat Oncol Biol Phys (2013) 86:469-76. doi:10.1016/j.ijrobp.2013.02.015

21. Owonikoko TK, Zhang G, Deng X, Rossi MR, Switchenko JM, Doho GH, et al. Poly (ADP) ribose polymerase enzyme inhibitor, veliparib, potentiates chemotherapy and radiation in vitro and in vivo in small cell lung cancer. Cancer Med (2014) 3(6):1579-94. doi:10.1002/cam4.317

Conflict of Interest Statement: The authors declare that the research was conducted in the absence of any commercial or financial relationships that could be construed as a potential conflict of interest.

Copyright (c) 2015 VanderWeele, Paner, Fleming and Szmulewitz. This is an openaccess article distributed under the terms of the Creative Commons Attribution License (CC BY). The use, distribution or reproduction in other forums is permitted, provided the original author(s) or licensor are credited and that the original publication in this journal is cited, in accordance with accepted academic practice. No use, distribution or reproduction is permitted which does not comply with these terms. 